\title{
Perceiving Spatially Inseparable Objects: Evidence for Feature-Based Object Selection Not Mediated by Location
}

\author{
Ronald Hübner and Gerriet Backer \\ Technische Universität Braunschweig
}

\begin{abstract}
In 4 experiments, stimulus elements were arranged into an LED-like array, and letters were defined within the array by feature similarity between the elements with respect to color and form. These stimuli allowed the display of a target and a distractor letter simultaneously at the same location. They were spatially inseparable but could be separated in feature space. Participants had to identify the letter on a prespecified feature dimension (color or form). As a result, the distractors produced specific compatibility effects. This showed that nontarget features could not be ignored at an early stage (i.e., that color and form were processed automatically and in parallel up to a high stage). The target was selected from the resulting objects according to the prespecified feature dimension. Results demonstrate that object selection is possible without selecting absolute spatial arrays.
\end{abstract}

Although it is widely acknowledged that attention plays a major role for the selection of relevant information from the environment, there is an ongoing dispute about the unit of selection. Some researchers assume that attention is purely space based, whereas others propose that objects are selected. A major problem in resolving this issue is that objects occupy a unique space, which makes it difficult to distinguish both accounts. As a consequence, almost all of the results interpreted in favor of object-based selection are confounded by location. Therefore, we used a new type of stimuli in the present research that avoided such a confounding.

The most prominent models of space-based selection are the spotlight metaphor (e.g., B. A. Eriksen \& Eriksen, 1974; B. A. Eriksen \& Hoffman, 1972; Posner, 1980; Posner, Snyder, \& Davidson, 1980) and its derivatives (C. W. Eriksen \& St. James, 1986; LaBerge, 1995). The spotlight model assumes that attention can be restricted to a contiguous region of the visual field, where information is processed preferentially. Additionally, it is hypothesized that the size of the spotlight cannot be reduced below a certain minimum. Support for such an account has been provided by targetflanker experiments, in which a target letter has to be identified in the presence of flanking letters. If the flankers were located close to the target, then the participants could not prevent the compatibility between distractors and target to affect target identification. However, by increasing the distance between target and distractors, the flanker effect could be reduced. It was assumed that the flankers were located outside the attentional spotlight (B. A. Eriksen \& Eriksen, 1974; B. A. Eriksen \& Hoffman, 1972).

Ronald Hübner and Gerriet Backer, Department of Psychology, Technische Universität Braunschweig, Braunschweig, Germany.

This research was supported by a research grant from the Deutsche Forschungsgemeinschaft. We would like to thank Dirk Vorberg and Mary Peterson for their valuable comments on an earlier version of this article.

Correspondence concerning this article should be addressed to Ronald Hübner, who is now at the Fachgnuppe Psychologie, Universität Konstanz, Postfach 5560, D-78457 Konstanz, Germany.
Although space-based models of attentional selection have been predominant during the past few decades, it has also been suggested that selection operates on objects rather than on space. Unfortunately, because objects usually occupy a unique space, it is difficult to distinguish between object-based and space-based selection. Duncan (1984) tried to circumvent this problem by superimposing two objects: an outline rectangle and a line. Each of the objects had two relevant attributes, and the participants had to judge two of the four attributes in succession on each trial. It turned out that performance was superior when the two attributes referred to one object rather than to two. This result was interpreted by Duncan as evidence for object-based selection. Recently, however, Vecera and Farah (1994) argued that space could still have been involved. If one abandons the notion of a spotlight and assumes that attention can be focused on grouped arrays, that is, exclusively on the space occupied by an object irrespective of its form, then a line superimposed on a rectangle can also be selected by means of spatial information (see also Kramer, Weber, \& Watson, 1997).

Another method to confirm object-based selection is to vary feature relations between the items in a display while preserving their spatial relations. It is thought that, if, for instance, feature similarity modulates object relations, and the selection efficiency varies accordingly, then this could be considered as evidence for object-based selection. Such a method has been applied by several researchers (e.g., Baylis \& Driver, 1992; Harms \& Bundesen, 1983; Kramer \& Jacobson, 1991). For example, Kramer and Jacobson (1991) used the target-flanker paradigm and varied the color similarity between target and distractors in one condition. As a result, when target and distractors were of the same color, the interference increased, which was interpreted in favor of object-based selection. However, in another experiment Kramer and Jacobson found that the spacing of the items modulated this effect. Therefore, they concluded that neither an object-based nor a space-based model could fully account for their data and proposed a grouping-strength model, in 
which the output of the grouping process are not discrete objects but are entities organized along a continuum of grouping strength.

The results of these experiments demonstrate that the spotlight model of attention is too simple to account for all the data. However, on the other hand, they provide no clear evidence that attentional selection is possible without spatial information. The grouped-array model in particular is extremely difficult to differentiate from object-based selection. Obviously, spatial relations as well as feature relations determine which items in the display are grouped and selected simultaneously. Thus, although one can argue that grouped items constitute an object and that objects are therefore selected, one can also claim that not objects per se but their spatial array is selected. In any case, the idea that objects and features actually affect spatial selection cannot be excluded.

Furthermore, in visual search, where features have been shown to affect attentional selection (e.g., Duncan \& Humphreys, 1989; Treisman, 1988; Treisman \& Gelade, 1980; Wolfe, 1994), there is evidence that the effects are mediated by location. For instance, Tsal and Lavie (1993) found that after the detection of a color target the participants were faster at detecting a subsequent item adjacent to the target than at detecting an item that was similar in color. This suggests that features as such cannot be selected abstractly, only indirectly by their location (see also Shih \& Sperling, 1996). These results again show that location plays a major role for selection. Features appear to merely modulate the efficiency of selection.

Given these results, it seems justified to assume that attentional selection is primarily space based, although not in a simple spotlight manner. What is selected are grouped arrays. Such a model can even explain the selection of superimposed objects by a space-based mechanism, at least if their arrays are sufficiently separated, which is usually the case with superimposed line drawings (e.g., Duncan, 1984; Neisser \& Becklen, 1975; Rock \& Guttman, 1981). In many studies concerned with object-based selection, because one object in the visual field had to be selected to the exclusion of another (e.g., Baylis \& Driver, 1992; Harms \& Bundesen, 1983; Kramer \& Jacobson, 1991), it is conceivable that not only the array of the target object was sampled in a preferred manner but also that the arrays of competing objects were suppressed. Although Neisser and Becklen (1975) did not see any reason to assume such a suppression, more recent data indicate that inhibitory processes play an important role for attentional selection (for an overview, see Milliken \& Tipper, 1998).

In any case, for showing that object selection is possible without absolute spatial information, one needs objects that cannot be separated by their spatial arrays. One possibility would be to superimpose the spatial arrays of two objects to a large extent. However, how can two objects share the same spatial array without one object occluding the other? This is possible with the new stimulus type introduced here, one that allows researchers to display two spatially inseparable objects simultaneously, which, nevertheless, can be perceived individually. To reduce the information available for spatial selection to a minimum, we arranged elements into an LED-like array (see Figure 1). Within this configuration. letters were defined by feature similarity between the elements with respect to color or form. By using two feature dimensions, up to two different letters can be presented simultaneously within one stimulus. Depending on the specific dimension that attention is focused on, one or the other letter can be segregated and selected. Moreover, analogous to the target-flanker paradigm, a target letter can be displayed along with a compatible or incompatible distractor letter. However, here the target is defined not by its location relative to that of the distractor but by its respective feature dimension.

If one presents such stimuli, how could an observer's visual system separate and select the target letter? One way would simply be to ignore a specific feature. For instance, when the target is defined by luminance and one of the lower right stimuli shown in Figure 1 is given, then it could be segregated by filtering out the black elements, or put differently, by selecting the white ones. In this case, grouping by proximity would be sufficient for obtaining the global letter form. The target feature merely serves to determine the spatial array that has to be selected from the display (cf. Shih \& Sperling, 1996; Tsal \& Lavie, 1993). If an observer's visual system proceeds in such a way, then there should be no specific effect of an object defined on the nontarget-feature dimension (form, in our example) because its array is "hidden behind" the target's array.

However, there is physiological (e.g., Livingstone \& Hubel, 1988; Zeki \& Shipp, 1988) as well as experimental (e.g., Boucart \& Humphreys, 1992; Humphreys \& Boucart, 1997; Treisman, 1988; Treisman \& Gelade, 1980) evidence that color and form dimensions are processed, at least to some extent, in separate pathways. Therefore, another way for separating the letters in our stimuli would be to select not a single feature but a feature dimension. Within each

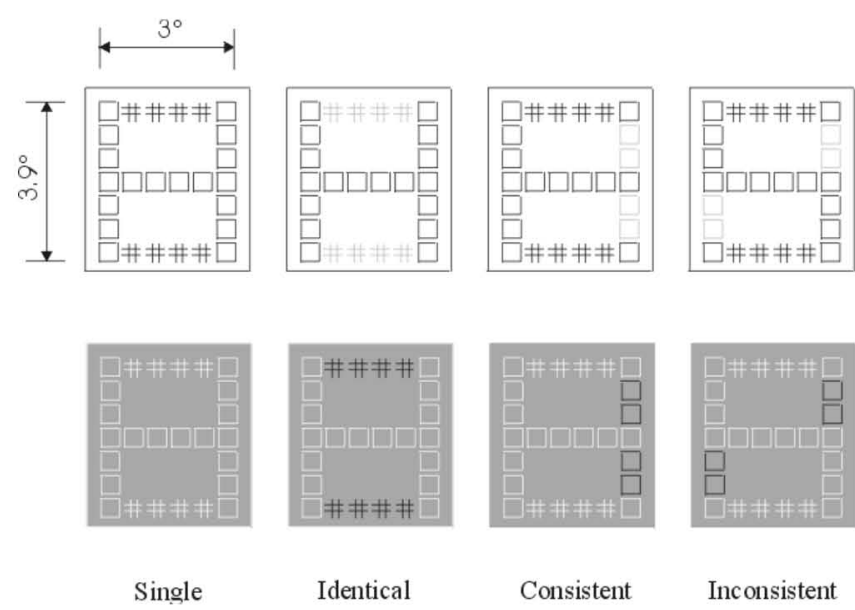

Figure 1. Examples of stimuli used in Experiment 1. Letters result from grouping by form and by color (luminance). If the square elements are grouped (grouping by form), then the result is an $\mathrm{H}$ in all cases. In the consistent and inconsistent stimuli a second letter ( $E$ or $S$ ) results from grouping by color. 
dimension, the items can then be grouped with respect to feature similarity. Applied to our example stimuli in the lower right portion of Figure 1, this means that, for selecting the luminance-defined letter, one would not select the white items but the complete color (luminance) information while ignoring form differences.

Alternatively, it is conceivable that, despite this controlled selection process, the information on the nontarget dimension is also automatically processed up to some degree in the form pathway. Unfortunately, the literature is inconclusive about how far form and color are processed independently and the extent to which people can access one information and ignore the other. For instance, Callaghan (1989) found that texture segregation on one dimension was impaired when a second irrelevant dimension varied, which contradicts a strong independence assumption. Humphreys and Boucart (1997) showed that there are independent processing pathways for form and color but that the activation within one pathway can influence the activation in the other (see also Boucart \& Humphreys, 1992: Cohen, 1997; Pashler, 1988). Taken together, in most experiments some kind of interaction between color and form pathways was found. However, it is not clear at what stage the interaction takes place. In any case, it cannot be ruled out that color and form are processed independently, at least at some stages. For instance, it could be possible that the objects in our stimuli were selected at a relatively late stage depending on their constituting features, irrespective of the absolute location of their spatial arrays. If this were the case, then one would expect the distractors to produce corresponding compatibility effects.

These considerations show that different selection processes predict different effects of the distractor letters in our stimuli. Because the objects are not spatially separable, feature processing is inevitable for their segregation and selection. If feature information merely determines the absolute spatial array that is selected, then there should be no specific distractor effects and we can conclude that the selection was space based mediated by features. On the other hand, if specific distractor effects occur, then this indicates that form and color information are processed in separate pathways up to an object stage and that the selection was object based mediated by features.

Four experiments were conducted to test these predictions. In the first experiment, additional to form, luminance was used as a feature dimension. In the other three experiments, hue was used instead. For convenience, both luminance and hue are regarded as a "color" dimension. Because it is known that the segregation of elements increases with their contrast ratio (Beck, Graham, \& Sutter, 1991), we used several luminance ratios in Experiment 1.

\section{Experiment 1}

\section{Method}

Participants. Six 20- to 27-year-olds (1 man and 5 women) participated in the experiment. All had normal or corrected-tonormal vision and participated either for course credit or for payment.
Apparatus. The stimuli were presented on a 19 -iri. $(48.26 \mathrm{~cm})$ color monitor with a resolution of 1,280 × 1,024 pixels, which was connected to a graphics board with 256 gray levels and a refresh rate of $75 \mathrm{~Hz}$ (noninterlaced). A PC controlled stimuli presentation and response registration.

Stimuli. The stimuli consisted of 26 elements that were arranged within a $6 \times 7$ grid so that the resulting global shape was similar to that of a figure eight (see Figure 1). At a viewing distance of $127 \mathrm{~cm}$, which was held constant by a chin rest, the global shape subtended a visual angle of $3.0^{\circ}$ horizontally and $3.6^{\circ}$ vertically. The elements subtended $0.41^{\circ} \times 0.42^{\circ}$, respectively.

Different element types were used to construct letters in the LED-like array. The element types differed with respect to their form, color, or both. With respect to form, squares and double crosses were used as elements (see Figure 1). The color of the elements was varied in three different contrast conditions, which are explained shortly

A target letter was defined by element similarity with respect to a given target dimension (color or form). With respect to a given dimension, the elements belonging to the letter or object and the remaining elements are denoted as foreground and background elements, respectively. For the form dimension, the squares were used as foreground elements and the double crosses as background elements. For instance, the squares in each of the bottom stimuli in Figure 1 can be grouped to result in the form of an $H$. In most conditions not only the target letter was present in a stimulus, but also a distractor letter defined by similarity with respect to the nontarget dimension. For instance, if the lower consistent stimulus in Figure 1 is grouped by color (luminance), then the result is an $\mathrm{E}$.

Four different letters, E, H, P, and S, were used and were mapped onto two response categories, $\{\mathrm{E}, \mathrm{H}\}$ and $\{\mathrm{P}, \mathrm{S}\}$.

There were three different contrast conditions (for examples, see Figure 1):

1. Low contrast: Foreground elements were displayed with a luminance of $82 \mathrm{~cd} / \mathrm{m}^{2}$ and the background elements with a lower luminance $\left(45 \mathrm{~cd} / \mathrm{m}^{2}\right)$ on a black screen $\left(0.314 \mathrm{~cd} / \mathrm{m}^{2}\right)$.

2. Medium contrast: The same luminances as in the low-contrast condition were used, except that the background elements had a luminance of $32 \mathrm{~cd} / \mathrm{m}^{2}$.

3. High contrast: Foreground elements were white $\left(82 \mathrm{~cd} / \mathrm{m}^{2}\right)$, whereas the background elements were black $\left(0.314 \mathrm{~cd} / \mathrm{m}^{2}\right)$ on a gray screen $\left(45 \mathrm{~cd} / \mathrm{m}^{2}\right)$.

For each of these contrast conditions, there were two target dimensions (color and form) and four consistency conditions:

1. Identical: The target letter was present on both dimensions (color and form).

2. Single: Only the target letter was presented (on the target dimension).

3. Consistent: A letter different from the target letter but belonging to the same response category was present on the nontarget dimension (i.e., the letter was response compatible).

4. Inconsistent: $A$ letter from the other response category was present on the nontarget dimension (i.e., it was response incompatible).

Examples of corresponding stimuli are shown in Figure 1.

Procedure. At the beginning of each trial, a cue appeared for $500 \mathrm{~ms}$ in the center of the screen. The cue consisted of the outline of a rectangle in a size that corresponded to that of the whole stimulus. After a blank interval of $600-900 \mathrm{~ms}$, the stimulus was presented for $160 \mathrm{~ms}$ and was followed by a blank screen. One thousand milliseconds after the response, the next trial began with the appearance of a cue. Response errors were signaled by a tone.

The color of the cue informed the participants of the target dimension: A white cue and a blue cue indicated form and color, respectively. The task of the participants was to classify the letter 
on the target dimension by pressing one out of two response buttons with their index or middle finger. respectively, of the same hand. The mapping of the letter pairs to the response keys (or fingers) was counterbalanced across subjects.

Altogether there were 24 conditions: three color conditions (low contrast. medium contrast, and high contrast), two target dimensions (color and form, describing the dimension through which the larger letter was defined), and four consistency conditions (identi. cal, single, consistent, and inconsistent).

The color conditions were blocked, where the sequence of blocks was counterbalanced between participants. All other conditions were randomized within each block. One block consisted of 64 trials. It look three 1-hr sessirns for each participant. Each block consisted of 64 stimuli; six blocks were carried out for training, and 30 blocks were carried out for the data. Altogether, there were 80 trials for each condition and participant, resulting in 2,304 trials for each participant.

\section{Results}

Response times (RTS). Only the latencies of correct responses were included in the data analysis. The mean latencies were entered into a three-factor analysis of variance (ANOVA) for repeated measurements on all factors: contrast (high, medium, and light), target dimension (color and form), and consistency (identical, single, consistent, and inconsistent).

Although the consistency factor was significant $(556,588$, 660 , and $712 \mathrm{~ms}), F(3,15)=10.9, p<.001$, neither the main effect of contrast nor that of target dimension turned out to be reliable. However, there was a significant interaction between the contrast factor and the target dimension factor, $F(2,10)=16.5, p<.001$. The results of this experiment are shown in Figure 2. It is obvious that the (RT) differences between the form and luminance dimensions decreased with increasing luminance. The performance for both dimensions was almost identical in the high-contrast (black-white) condition.

Furthermore, the interaction between the contrast factor and the consistency factor was reliable, $F(6,30)=5.39, p<$ .001 , whereas the interaction between the target dimension and the consistency factor was not, $F(3,15)=3.11, p=$ .058. However, there was also an interaction among all factors, $F(6,30)=4.06, p<.01$.

Because the black-white condition seemed to be the most interesting one for the present objective, we applied additional post hoc Bonferroni $t$ tests for paired comparisons to these data. We tested whether the mean latency difference between the single and the consistent condition, and that between the consistent and inconsistent conditions, was reliable. It turned out that the former difference $(118 \mathrm{~ms})$ was significant, $t(11)=3.86, p<.01$, as well as the latter $(64 \mathrm{~ms}), t(11)=5.38, p<.01$.

Error rates. The error rates are also given in Figure 2. Errors occurred, on average, in $14.2 \%$ of the trials. After an arcsin transformation, we submitted the error rates to a three-factor ANOVA analogous to that for the latency data. All main effects were significant: the effects of contrast, $F(2,10)=10.2, p<.01$, of target dimension, $F(1,5)=$ $9.25, p<.05$, and of consistency, $F(3,15)=42.5, p<.001$. In addition, the interactions between contrast and target

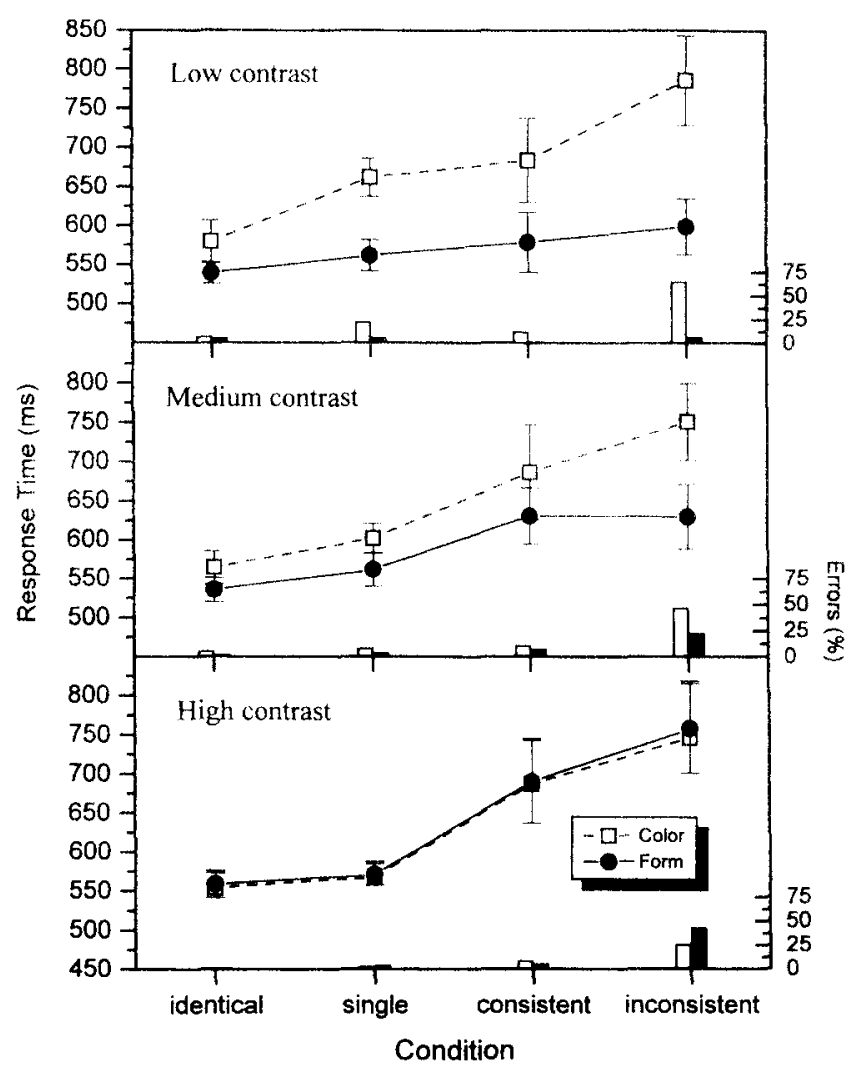

Figure 2. The results for the different conditions in Experiment 1. The error bars on the graphs indicate the standard error. The black and white vertical bars show the error rates with respect to the form and color dimension, respectively.

dimension, $F(2,10)=31.1, p<.001$, between contrast and consistency, $F(6,30)=4.83, p<.01$, and between target dimension and consistency, $F(3,15)=4.56, p<.05$, were reliable. Finally, the interactions among all three factors were significant, $F(6,30)=14.2, p<.001$. Altogether, the error rates varied in a way similar to the latencies, so that speed-accuracy trade-off effects can be excluded.

\section{Discussion}

First, our results show that the participants were able to select a letter defined on a prespecified feature dimension, even when it was superimposed by a different letter defined on a different feature dimension. Most important, however, the specific information on the nontarget dimension could not be ignored. The identity of the distractor letters produced specific effects. Incompatible distractors increased the latencies and error rates to a larger extent than did compatible ones. When the feature dimensions differed in saliency, as in the low-contrast condition, then it was not surprising that the information on the stronger dimension affected performance. However, even when both dimensions were similar in dominance, as in the black-white condition, the specific information on the nontarget dimension could not be ignored. 
Oul results show that object selection is possible without spatial information. The feature-mediated processes did not rely on the absolute location of the object's spatial array. Rather, the interference effects suggest that selection took place at a relatively late stage of processing. Thus, grouping hy sumilarity and object formation seems to have occurred in parallel on both feature dimensions. The target was then selected from the resulting objects according to the specified larget dimension. If the feature of the target had merely determined the spatial array to select, the remaining artay would not have been sufficient for the identification of the distractor letter and, consequently, there should have been no compatibility effects.

In aceord with Kramer and Jacobson's (1991) groupingstrength model, the efficiency of grouping and selection depended on the saliency or strength of the features. By varying the contrast (luminance differences) between the items in the display, the relative difficulty between color (luminance) selection and form selection could be modified. With the low-contrast stimuli, the selection of form was relatively easy, whereas the participants had great difficulties in color selection. The large error rate for color targets in the inconsistent condition indicated that the participants often either identified the letters on the nontarget (form) dimension or simply guessed. However, with increasing contrast, the color-selection performance improved, whereas the form-selection performance decreased. In the high-contrast (black-white) condition, the performance was similar for both dimensions, which indicates that color grouping and form grouping were equal in strength in this condition.

Although our results indicate that grouping proceeded automatically on individual feature dimensions, it was obvious that a pattern on the nontarget dimension also produced nonspecific costs. The fact that even consistent stimuli produced longer latencies than single-letter stimuli suggests that the processing on one dimension was affected by feature heterogeneity on the other dimension. However, does our heterogeneity effect simply reflect an increase in grouping difficulty, or was there a conflict at higher stages? The result that an identical letter on the nontarget dimension produced no latency increase, relative to the single-letter condition, suggests that grouping across heterogeneous elements is more difficult than grouping across homogenous ones. Yet, there could also be some selection conflict in the case of two different objects. It bears mention that in target-flanker experiments, compatible flankers also have a positive effect relative to neutral and incompatible flankers, but not relative to no flankers (e.g., B. A. Eriksen \& Eriksen, 1974), at least when the flankers are presented simultaneously with the target (cf. Grice \& Gwynne, 1985). This is the reason why compatibility effects are often defined relative to a neutral baseline condition.

Because no neutral objects were included in Experiment 1 , it is open whether a compatible but different letter on the nontarget dimensions produced faster responses than a neutral object. A neutral condition would also allow one to estimate the negative effect of heterogeneity. If simply grouping difficulty is increased with compatible distractors relative to a single-letter condition, then neutral distractors should have similar effects as compatible ones. This prediction was tested in a second experiment. To investigate the effect of heterogeneity in some detail, we included several neutral conditions that differed in this respect. Maximum heterogeneity was obtained by alternating the features of the nontarget dimension across elements. In two additional neutral conditions, heterogeneity was reduced by separating the elements into two contiguous regions, each with a different but homogeneous non-target-dimension feature. The two conditions differed with respect to the relative length of the two regions. Because the homogeneous regions had some objectlike character, we could regard them as neutral objects.

Because of the high error rates in the medium- and low-contrast conditions of Experiment 1, we retained only the black-white conditions and used an additional red-green condition.

\section{Experiment 2}

\section{Method}

Participants. Eight 19- to 27-year-olds (1 man and 7 women) participated in the experiment. All had normal or corrected-tonormal vision and participated either for course credit or for payment.

Stimuli. The stimuli were similar to those used in Experiment 1. However, instead of the low- and medium-contrast conditions, here a condition was used in which the foreground and background elements were red and green, respectively. Using a simple matching procedure, we adjusted the intensities of red and green so that they were roughly similar in luminance. However, we did not consider the objective that the elements were of equal luminance as crucial for the present experiment.

The same letters and categories used in Experiment 1 were used here. Concerning the neutral conditions, there were different structural arrangements on the nontarget dimension, which we describe next.

Conditions. Altogether there were 24 conditions: two color conditions (black-white, green-red) $\times$ two target dimensions (color and form) $\times$ six consistency conditions. The consistency conditions were as follows:

1. Single: Only the target letter was present. There was no pattern on the nontarget dimension.

2. Alternate: The features of the respective nontarget dimension alternated across elements

3. Neutral short: With respect to the nontarget dimension, the stimulus was divided into two contiguous regions of foreground and background elements, respectively. The regions were of the same length (i.e., there were as many foreground elements as there were background elements). Two structures of this type were used.

4. Neutral long: The same construction rule as in the neutralshort condition was used, except that the region containing foreground elements was extended, so that its length corresponded approximately to that of the letters. Two structures of this type were used.

5. Consistent: A letter different from the target letter but belonging to the same response category was present on the nontarget dimension.

6. Inconsistent: A letter from the other response category was present on the nontarget dimension.

Procedure. The procedure was the same as that used in Experiment 1 . The color conditions were blocked, and the order of 
blecks wis counterbatanced between subjects. All other conditions were randomized within each block. It took three to four 1-hr sessions for each participant. Each block consisted of 96 stimuli. Four blocks were carried out for training and 20 blocks for collecting the data. Altogether, there were 80 experimental trials for each condition and participant, resulting in 1,920 trials for each participant.

\section{Results}

RTs. The mean latencies of correct responses were subjected to a three-factor ANOVA for repeated measures on all factors: color (black white, red green), target dimension (color and form), and consistency (single, alternate, short. long, consistent, and inconsistent).

The results are given in Figure 3. Only the main effect of consistency was significant $F(5,35)=32.3, p<.001$. However, consistency interacted with the color factor, $F(5,35)=3.21, p<.05$, and the color factor interacted with the dimension factor, $F(1,7)=6.16, p<.05$. Finally, there was a significant three-way interaction among all factors, $F(5,35)=4.01, p<.01$.

To analyze the obvious difference between the alternate and long condition for the black-white data in more detail, we computed a post hoc ANOVA for these data with

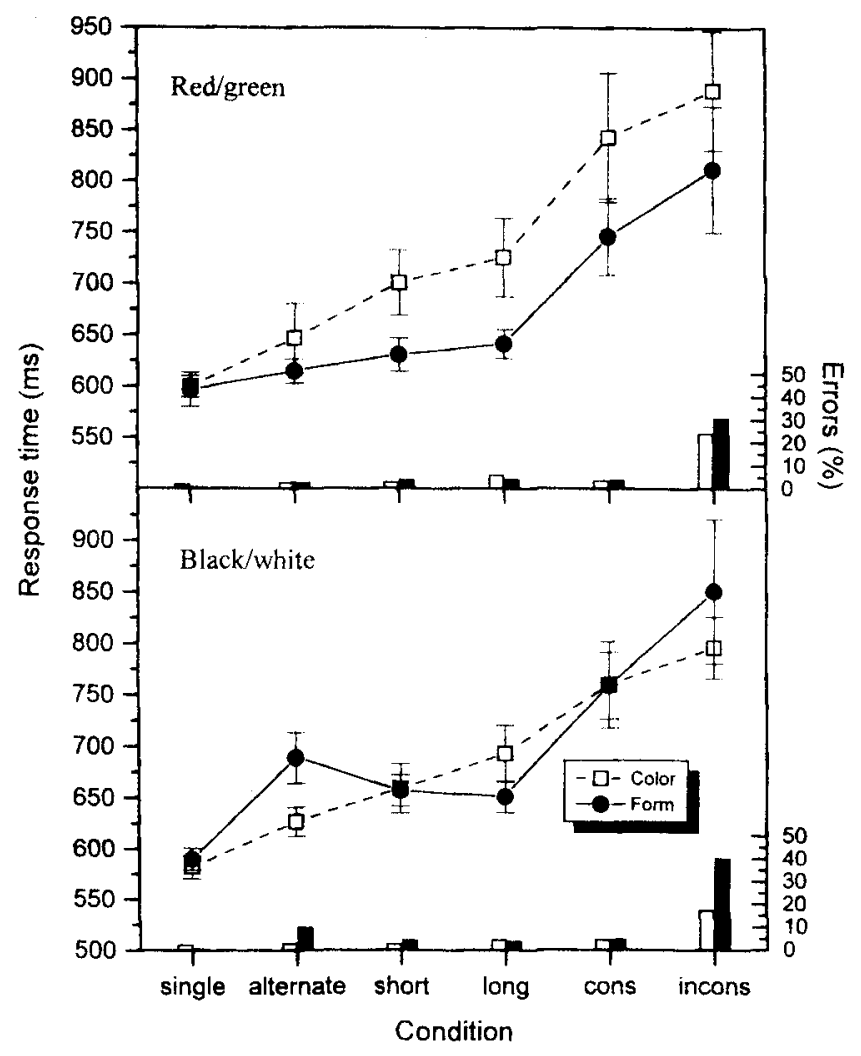

Figure 3. The results for the different conditions in Experiment 2. The error bars on the graphs indicate the standard error. The black and white vertical bars show the error rates with respect to the form and color dimension, respectively. cons = consistent; incons = inconsistent. condition (alternate and long) and target dimension as factors. Results show that the interaction between the two factors was significant. $F(1,7)=13.9, p<.01$. A similar analysis with the red-green data revealed only a significant condition effect, $F(1,7)=14.9, p<.01$.

For a more detailed analysis of the differences between the consistent, neutral, and inconsistent stimuli, we com puted two additional post hoc Bonferroni $t$ tests for paired comparisons. One test concerned the difference between the neutral-long and the consistent conditions $(98 \mathrm{~ms})$, which turned out to be significant, $t(31)=7.21, p<.001$. Furthermore, the difference between the consistent and inconsistent conditions $(60 \mathrm{~ms})$ was tested and found to be significant, $t(31)=3.65, p<.001$.

Errors. The error rates are also shown in Figure 3. Errors occurred, on average, in $8.09 \%$ of the trials. After an arcsin transformation, we subjected the error rates to a three-factor ANOVA analogous to that for the latency data. The main effects of color, $F(1,7)=9.46, p<.05$, and consistency, $F(5,35)=49.3, p<.001$, were significant Of the two-way interactions, that between consistency and target dimension, $F(5,35)=6.05, p<.001$, was significant. Finally, the interaction among all three factors was reliable, $F(5,35)=3.57, p<.05$. The error rates also varied in a way similar to the latencies, so speed-accuracy trade-off effects could be excluded.

\section{Discussion}

As in the first experiment, there were specific distractor effects. Also, the occurrence of a compatible but different letter on the nontarget dimension slowed the responses considerably relative to a single-letter stimulus. However. even compared with the neutral conditions, there was a latency increase for consistent stimuli. This is different from results observed in target-flanker experiments (e.g., B. A. Eriksen \& Eriksen, 1974) and shows that the performance reduction in the consistent condition, relative to the singleletter condition, does not rely exclusively on an increased grouping difficulty. Rather, what seems to matter is the presence of a response-relevant object on the nontarget dimension. However, how can the specific performance reduction for consistent objects be explained? Despite considerable saliency difference between color and shape in the red-green condition, the latencies between the neutrallong and the consistent conditions increased to the same extent for both dimensions, suggesting that some kind of selection conflict at a higher state of processing might have occurred not only in the inconsistent conditions but also in the consistent ones. We discuss this hypothesis in more detail later.

The results for the different neutral distractors show that there were nonspecific costs for grouping across different features on the nontarget dimension. Thus, the processing of color and form was not totally independent, which is in line with other results (e.g., Callaghan, 1989). Furthermore, there were some qualitative differences between the two color conditions. Grouping in the black-white conditions was different from that in the red-green conditions. When form 
features had to be grouped across elements with alternating contrast polarity, the performance was more impaired than in the alternating red-green condition. The difference between the two color conditions was less pronounced for neutral forms with larger contiguous regions of elements. That grouping across different directions of contrast is difficult has also been shown by visual search results (Treisman, 1993). On the other hand, there is evidence that attention can be directed selectively to elements of the same contrast polarity (Theeuwes \& Kooi, 1994).

Thus, the results of both experiments are consistent with the interpretation that grouping proceeds automatically and. to a large extent, independently on both feature dimensions and that a selection conflict occurs when two responserelevant but different objects are present. Because we used the same set of letters in both experiments, we tested the generality of these effects in a third experiment with a different set of letters and neutral forms.

\section{Experiment 3}

\section{Method}

Participants. Eight 16- to 32-year-olds ( 3 men and 5 women) participated in the experiment. All had normal or corrected-tonormal vision and participated either for course credit or for payment.

Stimuli. The stimuli were of the same type as those used in the previous experiments. However, this time the letters were $A, F, P$, and $S$. The letters $A$ and $F$ belonged to one response category and $P$ and $S$ to the other. Along with the letters, there were two neutral distractor forms that had the same extent as the letters. Only red-green was used as the color dimension. There were five different consistency conditions, depending on the forms present on the nontarget dimension.

Conditions. Altogether, there were 10 conditions: two stimulus dimensions (color and form) $\times$ five consistency conditions:

1. Identical: The target letter was present on both dimensions (color and form).

2. Single: Only the target letter was present. No pattern occurred on the nontarget dimension.

3. Neutral: A response-irrelevant structure (one of the two neutral forms, 1 ) was present on the nontarget dimension.

4. Consistent: A letter different from the target letter but belonging to the same response category was present on the nontarget dimension.

5. Inconsistent: A letter from the other response category was present on the nontarget dimension.

All conditions were randomized within each block, which comprised 80 trials. Two blocks were carried out for training and 8 blocks for collecting data. Altogether, there were 64 experimental trials for each condition and participant. The procedure was identical to that used in Experiment 1.

\section{Results}

RTs. The mean latencies of correct responses were subjected to a two-factor ANOVA for repeated measurements on all two factors: target dimension (color and form) and consistency (identical, single, neutral, consistent, and inconsistent).

The results are shown in Figure 4 . Only the consistency

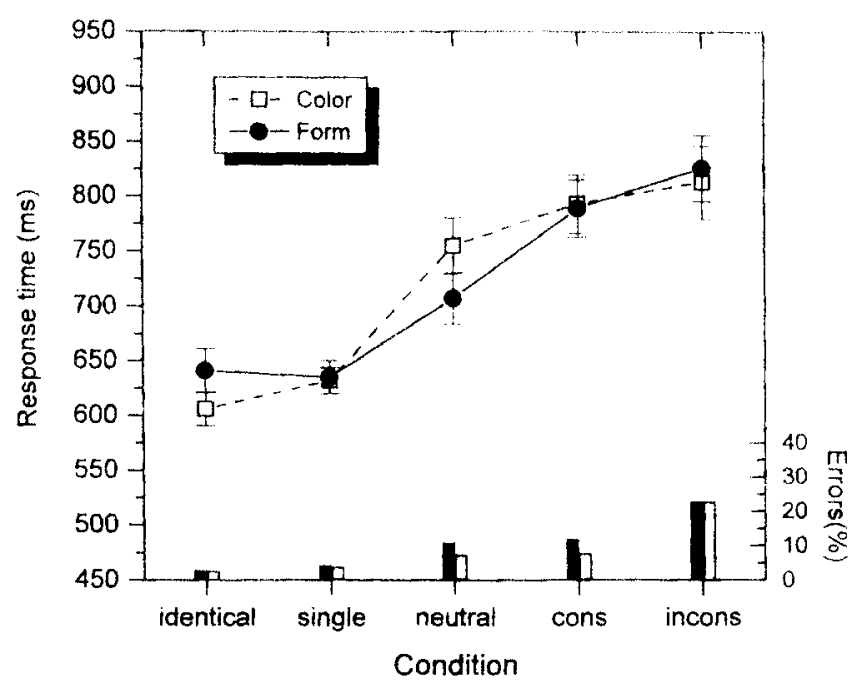

Figure 4. The results for the different conditions in Experiment 3 The error bars on the graphs indicate the standard error. The black and white vertical bars show the error rates with respect to the form and color dimension, respectively. cons = consistent; incons = inconsistent.

factor was significant, $F(4,28)=85.0, p<.001$. The interaction failed to reach significance, $F(4,28)=2.63, p<$ .056 .

To analyze the relevant consistency effects in more detail, we computed planned comparison between the neutral and the consistent condition and between the consistent and the inconsistent conditions. The former revealed a significant difference, $F(1,7)=27.1, p<.01$. The responses were faster $(731 \mathrm{~ms})$ when a neutral form was present on the nontarget dimension than when a compatible letter was present $(791 \mathrm{~ms})$. Furthermore, the latency difference between the consistent and inconsistent condition was significant, $F(1,7)=13.1, p<.01$. Responses to consistent stimuli were faster $(791 \mathrm{~ms})$ than those to inconsistent stimuli $(820 \mathrm{~ms})$.

Errors. The error rates are also shown in Figure 4. Errors occurred, on average, in $9.99 \%$ of the trials. After an arcsin transformation, only the mean error rates for the relevant consistency differences were analyzed. Although consistent stimuli produced fewer errors than inconsistent ones $(10.1 \%$ vs. $23.7 \%), F(1,7)=52.0, p<.001$, there was no reliable difference between the neutral and the consistent condition, $F(1,7)=1.74$. In any case, there was no indication of a speed-accuracy trade-off effect with regard to the relevant conditions.

\section{Discussion}

Compared with the first two experiments, the consistency effects here were smaller but reliable and point in the same direction as before. Again, consistent stimuli produced latencies that were increased relative to the neutral condition but that were faster than those to inconsistent stimuli. This 
shows that these effects occurred under different stimulus conditions.

Different from Experiment 2, however, the latencies for form and for color targets were similar. This could have been due to a learning effect. In Experiment 2 form remained constant throughout the blocks, whereas the color dimension varied (black-white vs, red-green). Thus, there were twice as many form trials as red-green trials. Here, both trial types were equal in number. However, there were also large individual differences. Although 3 of 8 participants had a color advantage in Experiment 2, their number increased to 5 in the present experiment. Therefore, it is possible that the mean difference between the latencies for the color and the form dimension was affected by the specific sample of participants.

\section{Experiment 4}

The results obtained in the last three experiments with our spatially inseparable letter objects demonstrate that the letters defined on the nontarget dimension were also processed. We interpreted the resulting consistency effect in the way that the participants were not able to ignore the information on the nontarget dimension. However, one might argue that the participants were in principle able to ignore the irrelevant letter but that randomizing the target dimensions encouraged them to encode the information on both dimensions on each trial. To test this objection that the consistency effect was merely a matter of task set, we conducted another experiment, in which a given target dimension remained constant throughout a block of trials. For comparison, we also included a condition with randomized target dimensions. Usually, consistency effects are increased in conditions in which attention has to be switched between stimulus dimensions across trials, relative to conditions with a constant dimension (e.g., Hübner, 1997). Thus, we predicted a reduction of the consistency effect under a constant target dimension. However, we expected it to still be significant.

We also tested whether the increased latencies for consistent stimuli relative to neutral ones were due to the fact that unknown shapes occurred on the nontarget dimension of neutral stimuli. One could argue that letters distract attention to a greater extent than do unknown shapes. Thus, here, letters were used as neutral shapes.

\section{Method}

Participants. Eight 22- to 43-year-olds (4 men and 4 women) participated in the experiment. All had normal or corrected-tonormal vision and participated either for course credit or for payment.

Stimuli. The stimuli were identical to those used in the previous experiment, except that the letters $\mathrm{L}$ and $\mathrm{U}$ were used as neutral distractors. Also, only stimuli corresponding to the neutral, consistent, and inconsistent conditions were presented. Thus, in this experiment two different letters always occurred in each stimulus, one on the target dimension and one on the nontarget dimension.
Conditions. There were 12 conditions total: two stimulus dimensions (color and form) $\times$ two block types (constant target dimension and randomized target dimension) $x$ three consistency conditions:

1. Neutral: A response-irrelevant letter (one out of the two neutral letters $U$ and $L$ ) was present on the nontarget dimension

2. Consistent: A letter different from the target letter but belonging to the same response category was present on the nontarget dimension.

3. Inconsistent: A letter from the other response category was present on the nontarget dimension.

The consistency conditions were randomized within each block In blocks with a constant target dimension, only one target dimension wecurred throughout the trials. The randomized blocks were analogous to those in the previous experiment. Four blocks (one for each target dimension and two with randomized target dimensions) were administered for training. Twenty blocks of 48 trials were then run in two 1 -hr sessions for collecting the data. Altogether, there were 80 experimental trials for each condition and participant

Procedure. The procedure was identical to that in Experiment 3. Here, however, blocks with a constant target dimension were also included. They alternated with blocks, in which the dimensions were randomized, where each participant started with a constant dimension. The target dimension (color or form) in the first block was balanced across participants.

\section{Results}

$R T s$. The mean latencies of correct responses were subjected to a three-factor ANOVA for repeated measurements on all factors: target dimension (color and form), block type (constant target dimension and randomized target dimension), and consistency (neutral, consistent, and inconsistent).

The results are shown in Figure 5. Block type had a significant effect, $F(1,7)=20.4, p<.01$. Responses were considerably faster in conditions with a constant target

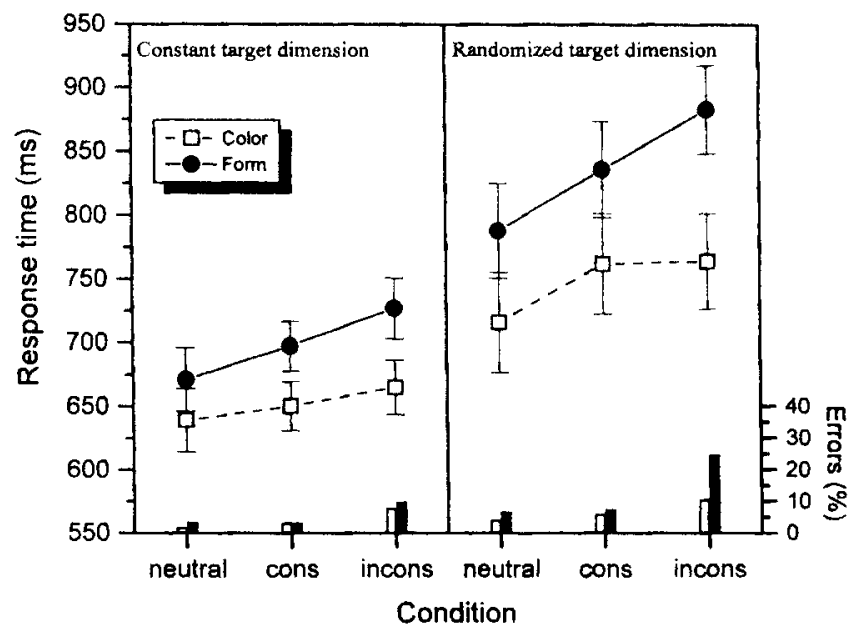

Figure 5. The results for the different conditions in Experiment 4. The error bars on the graphs indicate the standard error. The black and white vertical bars show the error rates with respect to the form and color dimension, respectively. cons $=$ consistent; incons $=$ inconsistent. 
dimension than in those with randomized dimensions $(675$ vs. $791 \mathrm{~ms})$. Also, the target dimension factor was significant, $F(1,7)=23.3, p<.01$. This time the participants responded faster to color-defined targets than to formdefined targets (699 vs. $767 \mathrm{~ms}$ ). A significant two-way interaction between block type and target dimension. $F(1,7) \cdots 12.9, p<.01$, indicated that the latency difference between the dimensions was smaller for the constant dimensions than for the randomized ones ( $47 \mathrm{vs} .88 \mathrm{~ms}$ ).

Furthermore, the consistency factor was significant, $F(2$, 14) $\cdots 59.3, p<.001$. The mean latencies for responses to neutral, consistent, and inconsistent stimuli were 704. 736, and $760 \mathrm{~ms}$, respectively. However, there was a reliable interaction between consistency and target dimension, $F(2$, $14)=8.40, p<.01$, reflecting that consistency affected form-defined targets more than color-defined targets. Obviously, under randomized target dimensions there was no difference between the RTs for consistent and inconsistent color-defined targets. A closer look at the data revealed that this was due to 2 participants, who were faster in the inconsistent than in the consistent color-target condition, and their other data were in the range of those of the other participants.

Finally, consistency and block type interacted significantly, $F(2,14)=6.80, p<.01$. To test whether the consistency effects were also reliable under constant target dimensions, we computed two planned pairwise comparisons. They indicated that the responses to neutral stimuli were significantly faster $(18 \mathrm{~ms})$ than those to consistent stimuli, $t(15)=2.47, p<.05$, and that the responses to consistent stimuli were faster $(22 \mathrm{~ms})$ than those to inconsistent stimuli, $t(15)=3.78, p<.01$.

Errors. The error rates are also shown in Figure 5. Errors occurred, on average, in $7.48 \%$ of the trials. After an arcsin transformation the mean error rates were analyzed analogous to the latencies. Block type had a significant effect, $F(1,7)=19.2, p<.01$. Fewer errors occurred with a constant target dimension than with randomized target dimensions $(5.0 \%$ vs. $9.9 \%$ ). Also, the target dimension factor was significant, $F(1,7)=14.7, p<.01$. Fewer errors were made with color-defined targets than with form-defined ones $(5.6 \%$ vs. $9.3 \%)$. Finally, the consistency factor was reliable, $F(2,14)=30.2, p<.001$. The error rates for neutral, consistent, and inconsistent stimuli were $4.1 \%$, $5.0 \%$, and $13.3 \%$, respectively.

Of the two-way interactions, those between block type and target dimension, $F(1,7)=8.56, p<.05$, and target dimension and consistency, $F(2,14)=6.46, p<.05$, were significant. The former interaction indicated that the error rate difference between the dimensions was smaller for constant target dimensions than for randomized dimensions, whereas the latter interaction indicated that there was a smaller consistency effect for color-defined targets than for formdefined ones. Because the error rates varied in the same way as the latencies, we excluded speed-accuracy trade-off effects.

\section{Discussion}

The results of this experiment demonstrate that the consistency effects observed in our previous experiments were not due solely to the randomization of the target dimension, which might have encouraged the participants to select information on both dimensions. Although, on average, the consistency effects were larger with randomized target dimensions than with constant ones, they were still reliable in the latter condition. Thus, even under a constant target dimension, inconsistent stimuli produced longer RTs than did consistent ones. Also, the result that neutral stimuli were processed faster than consistent ones was replicated under constant target dimensions. Moreover, this was the case even though letters were used as neutral forms here. Thus, the latter effect was not due to a larger distracting effect of known forms relative to unknown ones. A positive effect of the constant target dimensions was that the error rates were considerably reduced relative to the conditions with randomized dimensions in this and the previous experiments. This shows that the consistency effects as reflected by the latency data do not rely on high error rates.

Different from our previous experiments, here responses to color-defined targets were faster than those to formdefined ones. As mentioned before, there were large individual differences in this respect. Here, 7 of 8 participants had a color advantage. Thus, the participants in this experiment might represent an unbalanced sample. Yet, it cannot be excluded that also some experimental factors, such as the experience with a constant target dimension, were responsible for the appreciable color advantage.

\section{General Discussion}

The present research was concerned with the ongoing controversy of whether attentional selection is space or object based. Although there is ample evidence that object relations and feature relations between items in the visual field affect the efficiency of selection, this questions only the validity of simple space-based models, such as the spotlight metaphor, but not more sophisticated accounts of spatial selection. Unfortunately, the fact that selected items or configurations usually occupy a unique space makes it difficult to investigate object or object-based selection without confounding it by location. Particularly, the groupedarray hypothesis of spatial selection (Vecera \& Farah, 1994), which states that attention can be focused exclusively on the spatial array occupied by an object irrespective of its form, makes it difficult to demonstrate object-based selection that is not mediated by location. Thus, in most cases in which the effects of features or spatial arrangements were interpreted in favor of object selection (e.g., Baylis \& Driver, 1992; Duncan, 1984; Harms \& Bundesen, 1983; Kramer \& Jacobson, 1991), it can also be argued that the effects were due to variations of the efficiency with which the corresponding spatial arrays could be selected.

For not confounding object selection with location, in the present experiments, space was replaced by features as the basis for selection. The substitution was accomplished using LED-like arrays as stimuli, in which objects could be defined by means of feature similarity between the elements with respect to their color or form. In particular, these stimuli allowed us to display two spatially inseparable 
objects simultaneously within one array, which nevertheless could be perceived individually. Because spatial information was not sufficient for the selection of a certain object, the participants had to attend to a certain feature dimension for extracting the relevant object. Consequently, objects could merely be separated in feature space, but not in space. However, analogous to the target-flanker paradigm, interference effects could be investigated using compatible or incompatible distractors (c1. B. A. Eriksen \& Eriksen, 1974).

We hypothesized that. if the irrelevant feature or feature dimension could be ignored or filtered out at an early stage of processing. then there should be no specific distractor effects. On the other hand, the occurrence of such effects would indicate that the selection took place at a higher stage and consequently that in this case the information must have been processed on both dimensions simultaneously up to that stage.

All four experiments reported here clearly demonstrate specific distractor effects. When the object on the nontarget dimension was incompatible with the response to the target, performance was reduced relative to a compatible distractor. This effect shows that the selection was neither accomplished by filtering out a certain feature at an early stage nor by simply selecting the spatial array defined by the relevant feature (cf. Shih \& Sperling, 1996). As mentioned in the introduction, if a certain (background) feature could be filtered out, then the remaining elements could be grouped by proximity alone to give the shape of the target letter. In this case, the remaining information with respect to the nontarget dimension would be insufficient for the grouping of the distractor letter. For instance, if the selection of a color-defined target in the red-green condition had been accomplished by filtering out the green background elements, then the remaining red foreground elements would not have been sufficient for extracting the form-defined distractor. Consequently, there should have been no specific distractor effects. The same argument holds for the hypothesis that features determine the spatial array that is to be selected.

Our data are also incompatible with the notion that the information of a specific feature dimension or of a corresponding processing stream (e.g., Livingstone \& Hubel, 1988: Zeki \& Shipp, 1988) can be selected at an early stage. Also in this case one would have expected no compatibility effects. Thus, we can exclude that selection occurred early and that it was based on spatial information. Rather, our results indicate that the information was processed in parallel on the form and on the color dimension or in the respective pathways up to a high stage. This is consistent with the hypothesis that grouping proceeds preattentively (e.g., BenAv, Sagi, \& Braun, 1992; Enns \& Kingstone, 1995; Moore \& Egeth, 1997; Treisman, 1982), at least to some extent. In any case, following Kramer and Jacobson's (1991) groupingstrength model, we can even state that grouping occurs simultaneously on different feature dimensions.

The observed performance reduction under the neutral distractors relative to no distractors shows that there were also nonspecific effects produced by feature heterogeneity on the nontarget dimension. This indicates that the process- ing was not entirely independent in the different pathways (cf. Boucart \& Humphreys, 1992; Callaghan. 1989; Cohen. 1997: Humphreys \& Boucart, 1997; Pashler, 1988). How ever, the nonspecific effects were small relative to the compatibility effects. Therefore, it can be assumed that the processing on both dimensions proceeded not only in parallel but also to a large extent independently. This implies that the selection took place at a relatively late stage, where the output was even identified up to a certain degree. Attention merely served for the selection of an object according to the prespecified feature dimension, where this process was affected by the identity of the distractor.

Different from other results, here consistent stimuli pro duced slower responses than neutral ones. At first glance, this might seem to be a contradiction of otherwise similar results to those obtained with the target-flanker paradigm (e.g., B. A. Eriksen \& Eriksen, 1974). However, presenting a consistent stimulus meant, in our case, that two responserelevant objects had to appear at the same time at the same location. Thus, it is reasonable to assume that there was some kind of competition between response-relevant objects for being represented in visual short-term memory and for the control of behavior. For instance, if one assumes that objects are represented by object files (e.g., Kahneman \& Treisman, 1984), that is, by a temporary sensory representation of all the features present in a particular object, then several object files also must be created if several objects occur simultaneously. Moreover, each object file needs to be uniquely addressed in time and space. Our data suggest that it might not be possible to assign the same address to two object files without conflict. For consistent stimuli the resolution of this conflict consumes more time than the advantage produced by positive priming.

A representational account can also explain why stimuli containing two identical letters were processed considerably faster than two consistent but different letters. In this case, along with a nonspecific grouping advantage, the specification of a single letter (object file) is sufficient for a unique representation of the stimulus in visual memory. Interestingly, as the data of Experiment 4 demonstrate, familiarity of an object is obviously not sufficient for the creation of an object file. Even when letters instead of unknown shapes served as neutral distractors, they interfered less than compatible letters on the nontarget dimension. Thus, what seems to be important for the creation of an object file, at least for the superimposed objects used here, is that the respective object is response relevant.

A conceivable alternative account for our specific distractor effects would be to assume that the participants processed the two feature dimensions in succession. Assume, for instance, that the nontarget dimension was processed first on some trials, so that attention had to be switched to the other dimension. If the object on the nontarget dimension had already been identified, then compatibility effects would also be expected in this case. However, such an account is highly unlikely. It would also predict an advantage for consistent stimuli relative to neutral ones, which obviously was not the case. Thus, a simple attention-switching hypothesis does not hold, although one can think of additional 
assumptions that could account for the discrepancy. The main argument, however, is that attention switching predicts the performance on nonswitch trials to be similar in the different conditions. This implies, for instance, that the latencies of the fastest RTs in the neutral condition should be similar to those in the consistent condition. A reanalysis of our respective data revealed that this was not the case. Even the fastest latencies were shorter in the neutral condition than those in the consistent one. In view of these facts, we prefer a parallel account for our effects.

One of the reviewers was concerned about the fact that in former studies the absence of distractor effects was used to demonstrate object-based selection, whereas in our experiments the presence of such effects was used instead. Indeed, with spatially separable objects it is usually concluded that, if distractors do not produce interference, they are segregated from the target, which is selected and processed as a whole (e.g., Baylis \& Driver, 1992; Harms \& Bundesen, 1983; Neisser \& Becklen, 1975). As mentioned before, however, these resuits are also compatible with the hypothesis that objects affect only the segregation process and the efficiency with which certain spatial arrays are selected, that is, they do not show object-based selection but that objects can affect spatial selection (cf. Vecera \& Farah, 1997). On the other hand, in our experiments we observed featurebased object selection that does not rely on spatial selection. Nevertheless, there is a possible link between the two approaches that might explain the opposite effects. As mentioned in the introduction, there is evidence that spatial inhibition plays an important role in attentional selection. Thus, it is conceivable that object-based spatial selection proceeds not only by sampling the spatial array owned by the target object but also by inhibiting the arrays occupied by distractor objects. Such a procedure can even be applied to partially overlapping objects and could explain reduced distractor effects. However, this procedure cannot be applied to our stimuli. By placing the target into the same array as the distractor, we forced the visual system to refrain from object-based spatial inhibition. The spatial array of the distractor could not simply be inhibited because it was also occupied to a large extent by the target. Consequently, selection must be delayed to a later stage, at which it was based not on spatial information but on feature information and where, obviously, the identity of the distractor was known. Admittedly, this explanation of why consistency effects were observed here but not in other studies is highly speculative. Therefore, further research is needed to show whether it is valid.

In summary, several conclusions can be drawn from our results. First, object selection is possible that does not rely on absolute spatial arrays. Second, attention to features or feature dimensions does not simply determine the absolute spatial array to select. Third, form and color information cannot be ignored at an early stage of processing. Fourth, color and form can be processed (e.g., grouped) automatically and, to a large extent, independently up to a high stage, where attention to features can be used to select between the resulting objects.

\section{References}

Baylis, B. C., \& Driver, J. (1992). Visual parsing and response competition: The effect of grouping tactors. Perception \& Psychophysics, 51, 145-162.

Beck, J., Graham, N., \& Sutter, A. (1991). Lightness differences and the perceived segregation of regions and populations. Perception \& Psychophysics, 49, 257-269.

Ben-Av, M. B., Sagi, D., \& Braun, J. (1992). Visual attention and perceptual grouping. Perception \& Psychophysics, 52, 277-.294.

Boucart, M., \& Humphreys, G. W. (1992). Global shape cannot be attended without object identification. Journal of Experimental Psychology: Human Perception and Performance, 18. 785-806.

Callaghan, T. C. (1989). Interference and dominance in texture segregation: Hue, geometric form, and line orientation. Percep. tion \& Psychophysics, 46, 299-311.

Cohen, D. J. (1997). Visual detection and perceptual independence: Assessing color and form. Perception \& Psychophysics, 59. 623-635.

Duncan, J. (1984). Selective attention and the organization of visual information. Journal of Experimental Psychology: General, $113,501-517$.

Duncan, J., \& Humphreys, G. W. (1989). Visual search and stimulus similarity. Psychological Review, 96, 433-458.

Enns, J. T., \& Kingstone, A. (1995). Access to global and local properties in visual search for compound stimuli. Psychological Science, 6, 283-291.

Eriksen, B. A., \& Eriksen, C. W. (1974). Effects of noise letters upon the identification of a target letter in a nonsearch task. Perception \& Psychophysics, 16, 143-149.

Eriksen, B. A., \& Hoffman, J. E. (1972). Temporal and spatial characteristics of selective encoding form visual displays. Perception \& Psychophysics, 12, 201-204.

Eriksen, C. W., \& St. James, J. D. (1986). Visual attention within and around the field of focal attention: $A$ zoom lens model. Perception \& Psychophysics, 40, 225-240.

Grice, G. R., \& Gwynne, J. W. (1985). Temporal characteristics of noise conditions producing facilitation and interference. Perception \& Psychophysics, 37, 495-501.

Harms, L., \& Bundesen, C. (1983). Color segregation and selective attention in a nonsearch task. Perception \& Psychophysics, 33, $11-19$.

Hübner, R. (1997). The effect of spatial frequency on global precedence and hemispheric differences. Perception \& Psychophysics, 59, 187-201.

Humphreys, G. W., \& Boucart, M. (1997). Selection by color and form in vision. Journal of Experimental Psychology: Human Perception and Performance, 23, 136-153.

Kahneman, D., \& Treisman, A. (1984). Changing views of attention and automaticity. In R. Parasuraman \& D. R. Davies (Eds.), Varieties of attention (pp. 29-61). New York: Academic Press.

Kramer, A. F., \& Jacobson, A. (1991). Perceptual organization and focused attention: The role of objects and proximity in visual processing. Perception \& Psychophysics, 50, 267-284.

Kramer, A. F., Weber, T. A., \& Watson, S. E. (1997). Object-based attentional selection-grouped arrays or spatially invariant representations? Comment on Vecera and Farah (1994). Journal of Experimental Psychology: General, 126, 3-13.

LaBerge, D. (1995). Attentional processing: The brain's art of mindfulness. Cambridge, MA: Harvard University Press.

Livingstone, M., \& Hubel, D. (1988). Segregation of form, color, movement, and depth: Anatomy, physiology, and perception. Science, $240,740-479$. 
Milliken, B. \& Tipper, S. P. (1998). Attention and inhibition. In H Pashler (Ed.). Attention (pp. 191-221). Hove, England: Psychol ogy Press.

Moore, C. M. \& Egeth, H. (1997). Perception without attention Evidence of grouping under conditions of inattention. Journal of Experimental Psychologv: Human Perception and Performance. $23,339-352$

Neisser, U., \& Becklen, R. (1975). Selective looking: Attending to visually specified events. Cognitive Psychology, 7, 480-494.

Pashler, H. (1988). Cross-dimensional interaction and texture segregation. Perception \& Psychophysics, 43, 307-318.

Posner, M. I. (1980). Orienting of attention. Quarterly Journal of Experimental Pswchology, 32, 3-25.

Posner, M. I., Snyder, C. R. R., \& Davidson, B. J. (1980). Attention and the detection of signals. Journal of Experimental Psychol ogv: General. 109, 160-174.

Rock, I., \& Guttman, D. (1981). The effect of inattention on form perception. Journal of Experimental Psychology: Human Percep. tion and Performance, 7, 275-285.

Shih, S., \& Sperling, G. (1996). Is there feature-based attentional selection in visual search. Journal of Experimental Psychology: Human Perception and Performance, 22, 758-779.

Theeuwes, J., \& Kooi, F. L. (1994). Parallel search for a conjunction of contrast polarity and shape. Vision Research, 34, 3013-3016.

Treisman, A. (1982). Perceptual grouping and attention in visual search for features and for objects. Journal of Experimental Psychology: Human Perception and Performance, 8, 194-214.
Treisman, A. (1988). Features and objects: The 14th Bartlet Memorial Lecture. Quarterly Journat of Experimental Psychol. ogy: Human Experimental Psychology. 40A. 201 237.

Treisman, A. (1993). The perception of features and objects. In A Baddeley \& L. Weiskrantz (Eds.), Attention: Selection, aware ness and control (pp. 5-35). New York: Oxford University Press

Treisman, A., \& Gelade, G. (1980). A feature integration theory of attention. Cognitive Psychology, 12. 97-136.

Tsal, Y., \& Lavie, N. (1993). Location dominance in attending 10 color and shape. Journal of Experimental Psychology: Humam Perception and Performance, 19, 131-139.

Vecera. S. P.. \& Farah, M. J. (1994). Does visual attention select objects or locations. Antmal of Experimental Pswchology General. $123,146 \cdots 160$

Vecera, S.P. \& Farah, M. J, (1997) Is visual image segmentation a bottom-up or an interactive process? Perception \& Psychophys ics, 59, 1280-1296.

Wolfe, J. M. (1994): Guided Search 2.0: A revised model of visual search. Psychonomic Bulletin \& Review, 1, 202-238.

Zeki, S.. \& Shipp, S. (1988). The functional logic of cortical connections. Nature, 335. 311-317. 